CAHIERS DE

NARRATOLOGIE

\section{Cahiers de Narratologie}

Analyse et théorie narratives

$30 \mid 2016$

Street Art 2

\title{
Compte rendu. Ulrich Blanché, Banksy. Urban Art in a Material World
}

Edwige Comoy Fusaro

\section{(2) OpenEdition}

\section{Journals}

\section{Electronic version}

URL: http://journals.openedition.org/narratologie/7527

DOI: 10.4000/narratologie. 7527

ISSN: $1765-307 X$

Publisher

LIRCES

\section{Electronic reference}

Edwige Comoy Fusaro, « Compte rendu. Ulrich Blanché, Banksy. Urban Art in a Material World», Cahiers de Narratologie [Online], 30 | 2016, Online since 28 July 2016, connection on 19 April 2019. URL : http:// journals.openedition.org/narratologie/7527 ; DOI : 10.4000/narratologie.7527

This text was automatically generated on 19 April 2019

Article L.111-1 du Code de la propriété intellectuelle. 


\title{
Compte rendu. Ulrich Blanché, Banksy. Urban Art in a Material World
}

\author{
Edwige Comoy Fusaro
}

\section{REFERENCES}

Ulrich Blanché, Banksy. Urban Art in a Material World

trad. Rebekah Jonas \& Ulrich Blanché, Merburg, Tectum, 2016

1 Cet ouvrage rédigé en anglais est le premier volet d'une étude issue de la thèse de doctorat en histoire de l'art d'Ulrich Blanché, soutenue en 2012 à l'université de Heidelberg (Allemagne' ${ }^{1}$. Ce premier volet est la version anglaise remaniée du livre paru en langue allemande en 2012, Street Artivist Banksy. Konsumkunst. Kultur \& Kommerz bei Banksy and Hirst. Le second volet portera sur Damien Hirst et devrait paraître prochainement chez la même maison d'édition, également en anglais, sous le titre Damien Hirst. Gallery Art in a Material World. Le choix de la langue anglaise est parfaitement avisé, car le Street Art est un mouvement mondial qui impose l'emploi de la koiné du XXIe siècle. D'ailleurs, j'utiliserai ici les expressions Street Art, Urban Art, Style Writing, Graffiti et street artist sans italique, contre les normes typographiques en usage dans les publications en français et contre l'habitude nationale de franciser les mots et expressions créés dans la langue du ou des pays où l'objet désigné est d'abord apparu ou s'est d'abord développé (songeons à « ordinateur »).

2 Le parallélisme des titres et sous-titres précise l'approche privilégiée: d'une part, une comparaison entre l'un des plus grands street artists (sans doute le plus grand, si l'on tient compte du plus large spectre de critères possibles pour qualifier un artiste de " grand »), Banksy, et l'un des plus grands artistes contemporains, Hirst; d'autre part, le rapport de ces deux artistes à la société de consommation. Le matérialisme dont il est question dans ces titres s'entend au sens marxiste du terme, filtré par la culture de la pop music, étant entendu que le matérialisme « semble toujours représentatif de la société de consommation contemporaine ${ }^{2} »$. On comprend donc d'emblée trois choses. La première, 
c'est que le Street Art est traité sur un pied d'égalité vis-à-vis de l'art de galerie et qu'il constitue un mouvement à part entière de l'art contemporain: "Street Art pourrait bientôt devenir une expression historique, comme Young British $\mathrm{Art}^{3}$ ». On ne s'étonne donc guère que Banksy soit défini non seulement comme " un artiste politique ${ }^{4}$ », un " Street Artivist ", comme le qualifiaient les premières publications de Blanché, ou encore " un véritable enfant de son temps ${ }^{5}$ ", mais aussi comme "un véritable artiste moderne " tout court ${ }^{6}$. La deuxième chose que l'on remarque, c'est que l'art contemporain $-\mathrm{y}$ compris le Street Art - ne peut être bien appréhendé que par le dialogue polémique qu'il entretient avec la culture consumériste qui caractérise les sociétés des pays riches aujourd'hui. La troisième chose, c'est que la vision d'Ulrich Blanché est homogène à celle de son objet d'étude. Comme la plupart des œuvres de Banksy, le sous-titre de l'ouvrage est profondément sédimenté dans sa simplicité apparente. Dans le titre lui-même, le choix du seul pseudonyme de l'artiste ne renvoie pas à sa vie et/ou à sa personne, comme c'est le cas de nombreux autres ouvrages qui lui sont consacrés et comme pouvait le suggérer le titre de la version allemande - Street Artivist Banksy -, mais à sa persona au sens étymologique du terme, c'est-à-dire à son masque, à sa marque, à son œuvre au sens, accessoirement, des produits, et, surtout, au sens des stratégies opératoires, ambiguës, polémiques et géniales: à l'instar d'Andy Warhol, «Banksy» se lit et se mesure à l'enseigne du "principe d'aura ${ }^{7}$ », du motto "Des marques, pas des produits ${ }^{8}$ " qui caractérise une bonne part de l'art du XXe siècle. Blanché cible donc ici parfaitement l'art de Banksy, sur une ligne interprétative analogue à celle qu'a proposée Marie-Joseph Bertini dans le numéro précédent des Cahiers ${ }^{9}$. On voit bien qu'il propose ainsi une version plus mûre et une interprétation sensible et intelligente de l'œuvre de l'artiste.

Ce dernier aspect reflète la qualité de l'ensemble de l'étude. L'expression est claire, ainsi que la structure de l'ouvrage. La rigueur scientifique est presque impeccable. Aussi, notamment, Blanché définit-il systématiquement les termes qu'il emploie. Street Art désigne pour lui les «signes auto-autorisés dans l'espace public, souvent dans l'espace urbain, qui veulent communiquer avec un cercle plus large de spectateurs ${ }^{10} »-$ un public plus large, d'une part, que le cercle restreint et élitiste des consommateurs d'art de galerie et, d'autre part, que l'autre cercle encore plus restreint du Graffiti, que Blanché appelle Style Writing, et qui s'adresse in primis à ses propres membres -. Néanmoins, chacun sait qu'en marge des travaux réalisés dans la rue (synecdoque par laquelle je désigne l'espace public, notamment urbain), « auto-autorisés », c'est-à-dire non réalisés sur commande, et généralement illégaux et illicites ${ }^{11}$, les street artists produisent aussi des œuvres pour le commerce. C'est le cas de Banksy et c'est pourquoi Blanché parle d'Urban Art, qui regroupe à la fois le Street Art, le Style Writing « et l'art produit par des graffiti artists ou des street artists mais placé dans d'autres lieux et médias que la rue ${ }^{12} »$ : « rue » au sens précisé plus haut, ici aussi bien sûr. Blanché évoque les autres formes de créativité dans des espaces publics, telles que les « graffitis de salle de bain ${ }^{13}$ » (latrinalia), mais insiste à juste titre sur le fait que le Street Art n'est pas un art de galerie, de la même façon que le Street Art n'est pas le Style Writing, qu'on a coutume - à tort - de désigner simplement Graffiti (alors qu'il se caractérise par l'esthétique graphique, outre le cryptage du sens et la communication clanique). Blanché en vient alors inévitablement à la question : qu'est-ce que l'art ? Il n'y répond pas mais synthétise la problématique sous une forme interrogative: «L'art est-il l'idée ou l'exécution (simplement contrôlée par l'artiste $\left.{ }^{14}\right) ? »$. 
4 Le livre comprend sept points. Après l'introduction et l'élucidation des termes mis en présence (consommation et Street Art), le troisième point présente le contexte de la scène londonienne dans les vingt dernières années du XXe siècle, celles qui voient émerger à la fois Banksy et le mouvement Young British Art. Le chapitre 4 est consacré à Banksy. Plusieurs œuvres sont présentées et analysées, avec une attention marquée pour leur motivation, leur emplacement et leurs évolutions. Keep it Spotless (Defaced Hirst) fait l'objet d'une étude spécifique au point 5 : cette œuvre voit en effet collaborer les deux artistes étudiés et fait directement référence aux Pharmaceutical (Spot) Paintings de Hirst. Au sixième point, Blanché explique comment Banksy «montre de façon ironique ou parfois cynique les relations entre la consommation et la guerre, le nationalisme, la pollution, l'oppression, l'exploitation - c'est-à-dire entre la consommation et le pouvoir ${ }^{15}$ ». En dernière analyse, il conclut que, parce que c'est un art de l'hic et nunc et qu'il est " (im)matériel ", « l'art de Banksy peut être vu comme étant analogue à la vie humaine ${ }^{16}$ » : ce qui est valable pour le Street Art, à plus large échelle. Enfin la dernière section de l'ouvrage réunit des annexes très utiles sur la question du pseudonyme, les éléments biographiques, les expositions de l'artiste et la bibliographie.

Ulrich Blanché contextualise minutieusement les œuvres. De nombreux rapprochements sont établis, avec Koons, Hanson, Cattelan, Fleury et bien d'autres, qui donnent lieu à d'éclairantes considérations sur les sujets abordés par Banksy et la place de son œuvre au sein de l'art contemporain. On trouve en filigrane des éléments de reconstruction du mouvement du Street Art et une bibliographie sélective, certaines sources étant manifestement privilégiées : Naomi Klein, par exemple, ou Wolfgang Ullrich. Très attentif à la site-specificity, Blanché décrit scrupuleusement l'emplacement des œuvres. L'analyse des œuvres elles-mêmes est fine, faisant le jour sur leurs sédimentations sémantiques, connotatives et référentielles. Ceci est d'autant plus appréciable que, dans les études sur le Street Art, la description cède rarement la place au commentaire. Au demeurant, Ulrich Blanché affirme qu'il publie ici la première étude universitaire sur l'œuvre de Banksy (p. 13). Ce n'est pas tout à fait vrai, mais le fait est qu'il propose une monographie de grande qualité, qui se démarque des multiples autres livres déjà parus sur Banksy, ce dont on ne peut que se réjouir.

6 Enfin rien n'est spotless, pour autant que je sache, donc il faut aussi évoquer les faiblesses de l'ouvrage. Au plan formel, on relève de nombreuses coquilles et quelques omissions concernant les références. On rencontre parfois un mot allemand que l'auteur n'a pas jugé utile de traduire ou d'expliquer, ce qui est dommage. Il est également dommage que l'apparat illustratif, strictement limité aux œuvres faisant l'objet d'une étude approfondie, soit de piètre qualité. Il n'y a là rien de rédhibitoire, toutefois, puisqu'il est aisé de trouver d'autres images des œuvres sur internet. Au plan méthodologique, Blanché ne précise à aucun moment comment il a pu s'assurer que les œuvres dont il parle ont bien été réalisées par Banksy. Or, le succès dont l'artiste jouit lui vaut d'être largement copié, de manière avouée ou non, et quelles que soient les intentions du faussaire (notons au passage que la couverture du livre présente judicieusement le Food Art Work de Prudence Emma Staite, qui reproduit Keep it Spotless). Par ailleurs, Banksy luimême travaille beaucoup la question (et la valeur) de l'authenticité. À en juger par la qualité scientifique du travail, on ne doute guère que Blanché s'en soit systématiquement assuré, mais, s'agissant justement du fruit d'une recherche universitaire, on regrette de devoir se contenter de n'en pas douter. 


\section{NOTES}

1. Konsumkunst. Kultur \& Kommerz bei Banksy and Hirst

2. [« still seem representative of contemporary consumer societies »], p. 12.

3. [«Street Art could be a historical term soon, like Young British Art»], p. 11.

4. [« a political artist »], p. 129.

5. [« a true child of his time»], p. 131.

6. [« a true modern artist »], p. 132.

7. [« aura principle »], p. 198.

8. [« Brands Not Products»], p. 196.

9. Marie-Joseph Bertini, «Figures de l'anonymat. De quoi Banksy est-il le non?», Cahiers de Narratologie [En ligne], 29|2015, mis en ligne le 08 janvier 2016, consulté le 20 juin 2016. URL : http://narratologie.revues.org/7398

10. [" self-authorized signs in public space, often urban space that want to communicate with a larger circle of viewers »], p. 11.

11. [« non-legal, illicit or at least uncommissioned works »], p. 11.

12. [" art by Graffiti or Street Artists in other locations and media, than the street »], p. 12.

13. [« bathroom graffiti »], p. 91.

14. [« Is art the idea or the execution (only monitored by the artist)?»], p. 171.

15. [«shows ironically or sometimes cynically relationships between consumption and war, nationalism, pollution, oppression, exploitation - that is, between consumption and power », p. 190]

16. [« Banksy's art can be seen as analogous to human life »], p. 202. 